\title{
Anti-BCMA/CD3 BiTE Antibody REGN5458
}

National Cancer Institute

\section{Source}

National Cancer Institute. Anti-BCMA/CD3 BiTE Antibody REGN5458. NCI Thesaurus.

Code C158504.

A human bispecific T-cell engager (BiTE) antibody composed of two sing le-chain variable fragments (scFvs): one directed again the tumor-associated antigen (TAA) human B-cell maturation antigen (BCMA; tumor necrosis factor receptor superfamily member 17; TNFRSF17) and another directed against the CD3 antigen expressed on T-lymphocytes, with potential immunostimulating and antineoplastic activities. Upon administration, antiBCMA/anti-CD3 BiT E REGN5458 binds to both CD3 on cytotoxic T-lymphocytes (CT Ls) and BCMA on BCMA-expressing tumor cells. This activates and redirects CT Ls to BCMAexpressing tumor cells, leading to CT L-mediated killing of BCMA-expressing tumor cells. BCMA, a member of the tumor necrosis factor receptor superfamily that is specifically overexpressed on malignant plasma cells, plays a key role in promoting plasma cell survival. 